\title{
Chemical Reaction Effect on Transient Free Convective Flow past an Infinite Moving Vertical Cylinder
}

\author{
Ashish Paul ${ }^{1}$ and Rudra Kanta Deka ${ }^{2}$ \\ ${ }^{1}$ Department of Mathematical Sciences, Bodoland University, Kokrajhar 783370, India \\ ${ }^{2}$ Department of Mathematics, Gauhati University, Guwahati 781014, India
}

Correspondence should be addressed to Ashish Paul; ashpaul85@gmail.com

Received 18 March 2013; Accepted 17 August 2013

Academic Editor: Jose C. Merchuk

Copyright (C) 2013 A. Paul and R. K. Deka. This is an open access article distributed under the Creative Commons Attribution License, which permits unrestricted use, distribution, and reproduction in any medium, provided the original work is properly cited.

\begin{abstract}
An analysis is performed to study the heat and mass transfer on the flow past an infinite moving vertical cylinder, in the presence of first-order chemical reaction. The closed-form solutions of the dimensionless governing partial differential equations are obtained in terms of Bessel's functions and modified Bessel's functions by the Laplace transform technique. The transient velocity profiles, temperature profiles, and concentration profiles are studied for various sets of physical parameters, namely, the chemical reaction parameter, Prandtl number, Schmidt number, thermal Grashof number, mass Grashof number, and time. The skin friction, Nusselt number, and Sherwood number are also obtained and presented in graphs. It is observed that in presence of as well as increase in chemical reaction the flow velocity decreases. Also, in presence of destructive chemical reaction the concentration profile and Sherwood number tend to the steady state at large time.
\end{abstract}

\section{Introduction}

Combined heat and mass transfer in natural convection flows along moving vertical cylinders has got considerable attention in the last few decades because of its wide engineering and industrial application such as in hot rolling, hot extrusion, nuclear reactor cooling system, and underground energy system. Sparrow and Gregg [1] first studied the heat transfer from vertical cylinders. Goldstein and Briggs [2] presented an analysis of the transient free convective flow past vertical flat plate and circular cylinder for the unit and variable Prandtl number by employing the Laplace transform technique. Ishak [3] studied mixed convection over a vertical cylinder in presence of heat flux, while Lien et al. [4] studied the effects of free convection and mass transfer on the flow past an impulsively moving infinite vertical circular cylinder.

But, in nature, the presence of pure air or water is rather impossible. It is always possible that either foreign mass is present naturally in air or water, or foreign masses are mixed with air or water. A simple example is the naturally available water vapor that causes the flow of air. In many chemical engineering processes, there is chemical reaction between a foreign mass and the fluid in which the cylinder is moving. The rate of reaction, which is directly proportional to the concentration, is termed as first order chemical reaction. The effect of a chemical reaction depends on whether the reaction is homogeneous or heterogeneous. Chambré and Young [5] have analyzed a first order chemical reaction in the neighborhood of a horizontal plate. Das et al. [6] presented an analytical investigation of the effect of a homogeneous first order chemical reaction on the flow past an impulsively started infinite vertical plate with uniform heat flux and mass transfer by the usual Laplace transform technique. Muthucumaraswamy and Ganesan [7] studied the effect of firstorder chemical reaction on flow past an impulsively started vertical plate with uniform heat and mass flux by employing an implicit finite-difference scheme of the Crank-Nicolson type. Gangadhar et al. [8] studied hydromagnetic mixed convection heat and mass transfer over a vertical plate in the presence of magnetic field and chemical reaction. They have transformed the governing partial differential equations to a system of nonlinear differential equations and obtained the solution by using the Runge-Kutta-fourth order method with a shooting technique. 
Ganesan and Rani [9] presented a numerical solution of transient convection flow over a vertical cylinder under the combined buoyancy effects of heat and mass transfer in presence of first order chemical reaction. Again, Ganesan and Loganathan [10] investigated the combined effects of heat and mass transfer on a moving vertical cylinder under the assumption that chemically reactive species are emitted from the surface of the cylinder and are diffused into the fluid. Recently, Rani et al. [11] and Machireddy [12] studied the effect of chemical reaction on flow past over a vertical cylinder numerically under different physical situations.

However, closed-form solutions on natural convection along a moving vertical cylinder are very limited in the literature, which are very much necessary for validating numerical models. This may be due to the complicated mathematical approach involved in the analyses of such models. In the present paper, we have studied the combined buoyancy effects of heat and mass transfer from an infinite moving vertical cylinder in the presence of first order chemical reaction. The closed-form solutions of the system of nondimensional partial differential equations are obtained in terms of Bessel's functions and modified Bessel's functions by the Laplace transform technique. The effects of various physical parameters in the flow problem are discussed graphically.

\section{Mathematical Analysis}

Consider the unsteady, laminar, and incompressible viscous flow over an infinite vertical cylinder of radius $r_{0}$. Initially, it is assumed that the cylinder and fluid are at the same temperature $T_{\infty}^{\prime}$ and concentration $C_{\infty}^{\prime}$. At time $t^{\prime} \geq 0$, the cylinder starts to move in the vertical direction with constant velocity $u_{0}$, and the temperature and concentration near the cylinder are raised to constant temperature $T_{w}^{\prime}$ and concentration $C_{w}^{\prime}$, respectively. Here, the $x$-axis is taken vertically upward along the axis of the cylinder, and the radial coordinate $r$ is taken normal to the cylinder. The viscous dissipation is assumed to be negligible in the energy equation, as the motion is due to free convection only. It is also assumed that there exists a homogeneous first-order chemical reaction between the fluid and species concentration. Also, it is assumed that the level of species concentration is very low, and, hence, heat generated during chemical reaction can be neglected. Under these assumptions, the governing boundary-layer equations for momentum, energy, and concentration for free convective flow with Boussinesq's approximation are as follows:

$$
\begin{aligned}
& \frac{\partial u}{\partial t^{\prime}}= \frac{\nu}{r} \frac{\partial}{\partial r}\left(r \frac{\partial u}{\partial r}\right) \\
&+ g \beta\left(T^{\prime}-T_{\infty}^{\prime}\right)+g \beta^{*}\left(C^{\prime}-C_{\infty}^{\prime}\right) \\
& \frac{\partial T^{\prime}}{\partial t^{\prime}}=\frac{\alpha}{r} \frac{\partial}{\partial r}\left(r \frac{\partial T^{\prime}}{\partial r}\right) \\
& \frac{\partial C^{\prime}}{\partial t^{\prime}}=\frac{D}{r} \frac{\partial}{\partial r}\left(r \frac{\partial C^{\prime}}{\partial r}\right)-k_{1} C^{\prime}
\end{aligned}
$$

with the following initial and boundary conditions:

$$
\begin{aligned}
& t^{\prime} \leq 0: u=0, \quad T^{\prime}=T_{\infty}^{\prime}, \quad C^{\prime}=C_{\infty}^{\prime} \quad \forall r, \\
& t^{\prime}>0: u=u_{0}, \quad T^{\prime}=T_{w}^{\prime}, \quad C^{\prime}=C_{w}^{\prime} \quad \text { at } r=r_{0}, \\
& u \longrightarrow 0, \quad T^{\prime} \longrightarrow T_{\infty}^{\prime}, \quad C^{\prime} \longrightarrow C_{\infty}^{\prime} \quad \text { as } r \longrightarrow \infty .
\end{aligned}
$$

All the physical variables are mentioned in the Nomenclature section. In order to transform the governing equations into nondimensional form, we introduce the following dimensionless quantities:

$$
\begin{gathered}
R=\frac{r}{r_{0}}, \quad U=\frac{u}{u_{0}}, \quad T=\frac{T^{\prime}-T_{\infty}^{\prime}}{T_{w}^{\prime}-T_{\infty}^{\prime}}, \\
C=\frac{C^{\prime}-C_{\infty}^{\prime}}{C_{w}^{\prime}-C_{\infty}^{\prime}}, \\
\operatorname{Pr}=\frac{\nu}{\alpha}, \quad \mathrm{Sc}=\frac{\nu}{D}, \quad t=\frac{t^{\prime} \nu}{r_{0}^{2}}, \\
\mathrm{Gr}=g \beta r_{0}^{2} \frac{T_{w}^{\prime}-T_{\infty}^{\prime}}{u_{0} \nu}, \\
\mathrm{Gc}=g \beta^{*} r_{0}^{2} \frac{C_{w}^{\prime}-C_{\infty}^{\prime}}{u_{0} v}, \quad K=\frac{k_{1} r_{0}^{2}}{\nu},
\end{gathered}
$$

which reduces the governing equations (1) into

$$
\begin{gathered}
\frac{\partial U}{\partial t}=\frac{\partial^{2} U}{\partial R^{2}}+\frac{1}{R} \frac{\partial U}{\partial R}+\operatorname{Gr} T+\operatorname{Gc} C, \\
\frac{\partial T}{\partial t}=\frac{1}{\operatorname{Pr}}\left(\frac{\partial^{2} T}{\partial R^{2}}+\frac{1}{R} \frac{\partial T}{\partial R}\right), \\
\frac{\partial C}{\partial t}=\frac{1}{S c}\left(\frac{\partial^{2} C}{\partial R^{2}}+\frac{1}{R} \frac{\partial C}{\partial R}\right)-K C,
\end{gathered}
$$

with the following initial and boundary conditions:

$$
\begin{aligned}
& t \leq 0: U=0, \quad T=0, \quad C=0 \quad \forall R, \\
& t>0: U=1, \quad T=1, \quad C=1 \quad \text { at } R=1, \\
& U \longrightarrow 0, \quad T \longrightarrow 0, \quad C \longrightarrow 0 \quad \text { as } R \longrightarrow \infty .
\end{aligned}
$$

\section{Solution Technique}

To solve the governing nondimensional unsteady equations (4) subject to initial and boundary conditions (5), we apply the Laplace transform technique.

The Laplace transformation of (4) subject to initial conditions in (5) gives the following:

$$
\begin{gathered}
\frac{d^{2} \bar{U}}{d R^{2}}+\frac{1}{R} \frac{d \bar{U}}{d R}-p \bar{U}+\operatorname{Gr} \bar{T}+\operatorname{Gc} \bar{C}=0, \\
\frac{d^{2} \bar{T}}{d R^{2}}+\frac{1}{R} \frac{d \bar{T}}{d R}-p \operatorname{Pr} \bar{T}=0, \\
\frac{d^{2} \bar{C}}{d R^{2}}+\frac{1}{R} \frac{d \bar{C}}{d R}-\operatorname{Sc}(p+K) \bar{C}=0,
\end{gathered}
$$


where $p$ is the parameter of the Laplace transformation defined by $L\{f(t)\}=F(p)$, with $L$ being the Laplace operator, and $\bar{U}(R, p), \bar{T}(R, p)$, and $\bar{C}(R, p)$ are the Laplace transforms of $U(R, t), T(R, t)$, and $C(R, t)$ respectively.

Solutions of (7) and (8) subject to the transformed boundary conditions (5) give the following:

$$
\begin{gathered}
\bar{T}=\frac{K_{0}(R \sqrt{p \operatorname{Pr}})}{p K_{0}(\sqrt{p \operatorname{Pr}})}, \\
\bar{C}=\frac{K_{0}(R \sqrt{\mathrm{Sc}(p+K)})}{p K_{0}(\sqrt{\mathrm{Sc}(p+K)})} .
\end{gathered}
$$

Substituting the expressions of $\bar{T}$ and $\bar{C}$ in (6) and solving, the expression of $\bar{U}$ can be obtained as following:

$$
\begin{aligned}
\bar{U}= & \frac{K_{0}(R \sqrt{p})}{p K_{0}(\sqrt{p})}+\frac{\mathrm{Gc}}{p\{p(1-\mathrm{Sc})-K\}} \\
& \times\left\{\frac{K_{0}(R \sqrt{\mathrm{Sc}(p+K)})}{K_{0}(\sqrt{\mathrm{Sc}(p+K)})}-\frac{K_{0}(R \sqrt{p})}{K_{0}(\sqrt{p})}\right\} \\
& +\frac{\mathrm{Gr}}{p^{2}(1-\operatorname{Pr})}\left\{\frac{K_{0}(R \sqrt{p \operatorname{Pr}})}{K_{0}(\sqrt{p \operatorname{Pr}})}-\frac{K_{0}(R \sqrt{p})}{K_{0}(\sqrt{p})}\right\} .
\end{aligned}
$$

Using the theorem of the inverse Laplace transform on (9)-(10), it can be readily shown that (cf. Carslaw and Jaeger [13])

$$
\begin{gathered}
C=e^{-K t} \frac{1}{2 \pi i} \int_{\gamma-i \infty}^{\gamma+i \infty} e^{p t} \frac{K_{0}(R \sqrt{\mathrm{Sc} p})}{(p-K) K_{0}(\sqrt{\mathrm{Sc} p})} d p, \\
\times \int_{\gamma-i \infty}^{\gamma+i \infty} e^{p t}\left[\frac{K_{0}(R \sqrt{p})}{p K_{0}(\sqrt{p})}+\frac{\mathrm{Gc}}{p\{p(1-\mathrm{Sc})-K\}}\right. \\
\times\left\{\frac{K_{0}(R \sqrt{\mathrm{Sc}(p+K)})}{K_{0}\left(\sqrt{\mathrm{Sc}(p+K)}-\frac{K_{0}}{K_{0}(\sqrt{p})}\right)}\right\} \\
+\frac{\mathrm{Gr}}{p^{2}(1-\operatorname{Pr})\left\{\frac{K_{0}(R \sqrt{p \operatorname{Pr}})}{K_{0}(\sqrt{p \operatorname{Pr}})}\right.} \\
\left.T=\frac{1}{2 \pi i} \int_{\gamma-i \infty}^{\gamma+i \infty} \frac{K_{0}\left(R \sqrt{p} \frac{K_{0}(R \sqrt{p \operatorname{Pr}})}{p K_{0}(\sqrt{p \operatorname{Pr}})} d p .\right.}{K_{0}(\sqrt{p})}\right\} d p,
\end{gathered}
$$

Now, the integrand of (11) has a branch point at $p=0$ and a simple pole at $p=K$. Also, $K_{0}(\sqrt{\operatorname{Sc} p})$ do not have zero at

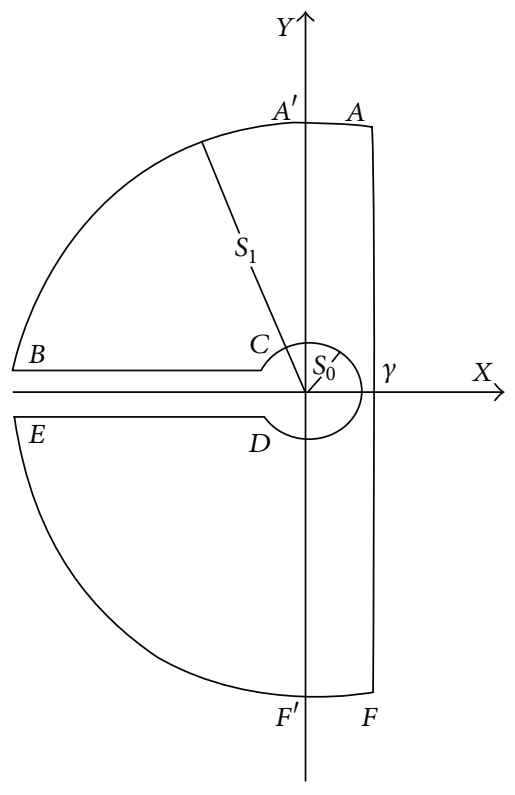

FIGURE 1: Contour for the inverse integral.

any point in the real and imaginary axes, if the branch cut is made along the negative real axis. To obtain $C(t, R)$ from $\bar{C}(p, R)$, we use the adjoining contour shown in Figure 1 . Accordingly, the line integral in (11) may be replaced by the limit of the sum of the integrals over FE, ED, DC, CB, and BA as $S_{1} \rightarrow \infty$ and $S_{0} \rightarrow 0$. It can be easily shown that the values of the integral (11) along the paths DC, BA, and FE approache zero as $S_{1} \rightarrow \infty$ and $S_{0} \rightarrow 0$.

Along the paths CB and ED, we choose $p=V^{2} e^{i \pi} / \mathrm{Sc}$ and $p=V^{2} e^{-i \pi} / \mathrm{Sc}$, respectively. Then we consider the following:

The line integral along

$$
\mathrm{CB}=e^{-K t} \frac{1}{\pi i} \int_{0}^{\infty} e^{-V^{2} t / \mathrm{Sc}} \frac{J_{0}(R V)-i Y_{0}(R V)}{\left(V^{2}+\mathrm{Sc} K\right)\left\{J_{0}(V)-i Y_{0}(V)\right\}} V d V,
$$

and the line integral along

$$
\mathrm{ED}=e^{-K t} \frac{1}{\pi i} \int_{0}^{\infty} e^{-V^{2} t / \mathrm{Sc}} \frac{J_{0}(R V)+i Y_{0}(R V)}{\left(V^{2}+\mathrm{Sc} K\right)\left\{J_{0}(V)+i Y_{0}(V)\right\}} V d V .
$$

Now, the residue of the integrand of (11) at $p=K$ is

$$
e^{K t} \frac{K_{0}(R \sqrt{K \mathrm{Sc}})}{K_{0}(\sqrt{K \mathrm{Sc}})} \text {. }
$$

Thus, using (14), (15), and (16) and applying theory of residues, we have the Laplace inverse of $\bar{C}$, which is the concentration given as

$$
\begin{aligned}
C= & e^{K t} \frac{K_{0}(R \sqrt{K \mathrm{Sc}})}{K_{0}(\sqrt{K \mathrm{Sc}})} \\
& +\frac{2 e^{-K t}}{\pi} \int_{0}^{\infty} \frac{e^{-V^{2} t / \mathrm{Sc}}}{\left(V^{2}+\mathrm{Sc} K\right)} \Gamma(R, V) V d V \quad(\text { for } K \neq 0) .
\end{aligned}
$$


In absence of chemical reaction $(K=0)$, it can be readily shown that

$$
C=1+\frac{2}{\pi} \int_{0}^{\infty} e^{-V^{2} t / \mathrm{Sc}} \Gamma(R, V) \frac{d V}{V},
$$

where

$$
\Gamma(R, V)=\frac{J_{0}(R V) Y_{0}(V)-Y_{0}(R V) J_{0}(V)}{J_{0}^{2}(V)+Y_{0}^{2}(V)} .
$$

Similarly, we obtain the expressions for velocity and temperature from (12) and (13) for $K \neq 0$ and $K=0$, respectively, as follows:

$$
\begin{aligned}
& U=1+\frac{2}{\pi} \int_{0}^{\infty} e^{-V^{2} t} \Gamma(R, V) \frac{d V}{V}+\frac{\mathrm{Gc}}{K \mathrm{Sc}}\left\{1-\frac{K_{0}(R \sqrt{K \mathrm{Sc}})}{K_{0}(\sqrt{K \mathrm{Sc}})}\right\} \\
& +\frac{2 \operatorname{PrGr}}{(\operatorname{Pr}-1) \pi} \int_{0}^{\infty}\left(1-e^{-t V^{2} / \operatorname{Pr}}\right) \\
& \times\left\{\Gamma\left(R, \frac{V}{\sqrt{\operatorname{Pr}}}\right)-\Gamma(R, V)\right\} \frac{d V}{V^{3}} \\
& -\frac{2 \mathrm{Gc}}{\pi} \int_{0}^{\infty}\left\{\frac{V e^{-\left(V^{2} / \mathrm{Sc}+K\right) t}}{\left(V^{2}+K \mathrm{Sc}\right)\left(\left(V^{2} / \mathrm{Sc}+K\right)(1-\mathrm{Sc})+K \mathrm{Sc}\right)}\right. \\
& \left.-\frac{e^{-V^{2} t}}{V\left(V^{2}(1-\mathrm{Sc})+K \mathrm{Sc}\right)}\right\} \Gamma(R, V) d V \\
& U=1+\frac{2}{\pi} \int_{0}^{\infty} e^{-V^{2} t} \Gamma(R, V) \frac{d V}{V} \\
& +\frac{2 \mathrm{PrGr}}{(\operatorname{Pr}-1) \pi} \int_{0}^{\infty}\left(1-e^{-V^{2} t / \mathrm{Pr}}\right) \\
& \times\left\{\Gamma\left(R, \frac{V}{\sqrt{\operatorname{Pr}}}\right)-\Gamma(R, V)\right\} \frac{d V}{V^{3}} \\
& +\frac{2 \mathrm{GcSc}}{(\mathrm{Sc}-1) \pi} \int_{0}^{\infty}\left(1-e^{-V^{2} t / \mathrm{Sc}}\right) \\
& \times\left\{\Gamma\left(R, \frac{V}{\sqrt{\mathrm{Sc}}}\right)-\Gamma(R, V)\right\} \frac{d V}{V^{3}}, \\
& T=1+\frac{2}{\pi} \int_{0}^{\infty} e^{-t V^{2} / \operatorname{Pr}} \Gamma(R, V) \frac{d V}{V} .
\end{aligned}
$$

Knowing the velocity, temperature, and concentration field deduced above, we now determine the skin friction $(\tau)$, Nusselt number $(\mathrm{Nu})$, and Sherwood number (Sh). In nondimensional form, the skin friction and Sherwood number for $K \neq 0$ and $K=0$, respectively, are obtained as follows:

$$
\begin{aligned}
& \left.\tau=-\frac{\partial U}{\partial R}\right]_{R=1} \\
& =\frac{2}{\pi} \int_{0}^{\infty} e^{-V^{2} t} \Gamma_{1}(V) d V-\frac{\mathrm{Gc}}{\sqrt{K \mathrm{Sc}}} \frac{K_{1}(\sqrt{K \mathrm{Sc}})}{K_{0}(\sqrt{K \mathrm{Sc}})} \\
& +\frac{2 \mathrm{PrGr}}{(\operatorname{Pr}-1) \pi} \\
& \times \int_{0}^{\infty}\left(1-e^{-V^{2} t / \operatorname{Pr}}\right)\left\{\frac{1}{\sqrt{\operatorname{Pr}}} \Gamma_{1}\left(\frac{V}{\sqrt{\operatorname{Pr}}}\right)\right. \\
& \left.-\Gamma_{1}(V)\right\} \frac{d V}{V^{2}} \\
& +\frac{2 \mathrm{Gc}}{\pi} \int_{0}^{\infty}\left\{\frac{V^{2} e^{-V^{2} t / \mathrm{Sc}-K t}}{\left(V^{2}+K \mathrm{Sc}\right)\left\{\left(V^{2} / \mathrm{Sc}+K\right)(1-\mathrm{Sc})+K \mathrm{Sc}\right\}}\right. \\
& \left.-\frac{e^{-V^{2} t}}{\left\{V^{2}(1-\mathrm{Sc})+K \mathrm{Sc}\right\}}\right\} \Gamma_{1}(V) d V \\
& (K \neq 0), \\
& \tau=\frac{2}{\pi} \int_{0}^{\infty} e^{-V^{2} t} \Gamma_{1}(V) d V \\
& +\frac{2 \mathrm{PrGr}}{(\operatorname{Pr}-1) \pi} \int_{0}^{\infty}\left(1-e^{-V^{2} t / \operatorname{Pr}}\right) \\
& \times\left\{\frac{1}{\sqrt{\operatorname{Pr}}} \Gamma_{1}\left(\frac{V}{\sqrt{\mathrm{Pr}}}\right)-\Gamma_{1}(V)\right\} \frac{d V}{V^{2}} \\
& +\frac{2 \mathrm{GcSc}}{(\mathrm{Sc}-1) \pi} \\
& \times \int_{0}^{\infty}\left(1-e^{-V^{2} t / \mathrm{Sc}}\right)\left\{\frac{1}{\sqrt{\mathrm{Sc}}} \Gamma_{1}\left(\frac{V}{\sqrt{\mathrm{Sc}}}\right)-\Gamma_{1}(V)\right\} \frac{d V}{V^{2}} \\
& (K=0) \text {, }
\end{aligned}
$$

where

$$
\begin{gathered}
\Gamma_{1}(V)=\frac{J_{1}(V) Y_{0}(V)-Y_{1}(V) J_{0}(V)}{J_{0}^{2}(V)+Y_{0}^{2}(V)}, \\
\left.\mathrm{Nu}=-\frac{\partial T}{\partial R}\right]_{R=1}=\frac{2}{\pi} \int_{0}^{\infty} e^{-V^{2} t / \mathrm{Pr}} \Gamma_{1}(V) d V, \\
\left.\mathrm{Sh}=-\frac{\partial C}{\partial R}\right]_{R=1}=\frac{\sqrt{K \mathrm{Sc}} K_{1}(\sqrt{K \mathrm{Sc}})}{K_{0}(\sqrt{K \mathrm{Sc}})} \\
+\frac{2 e^{-K t}}{\pi} \int_{0}^{\infty} \frac{e^{-V^{2} t / \mathrm{Sc}}}{V^{2}+K \mathrm{Sc}} \Gamma_{1}(V) V^{2} d V \\
\mathrm{Sh}=\frac{2}{\pi} \int_{0}^{\infty} e^{-\left(V^{2} t\right) / \mathrm{Sc}} \Gamma_{1}(V) d V \quad(K \neq 0),
\end{gathered}
$$




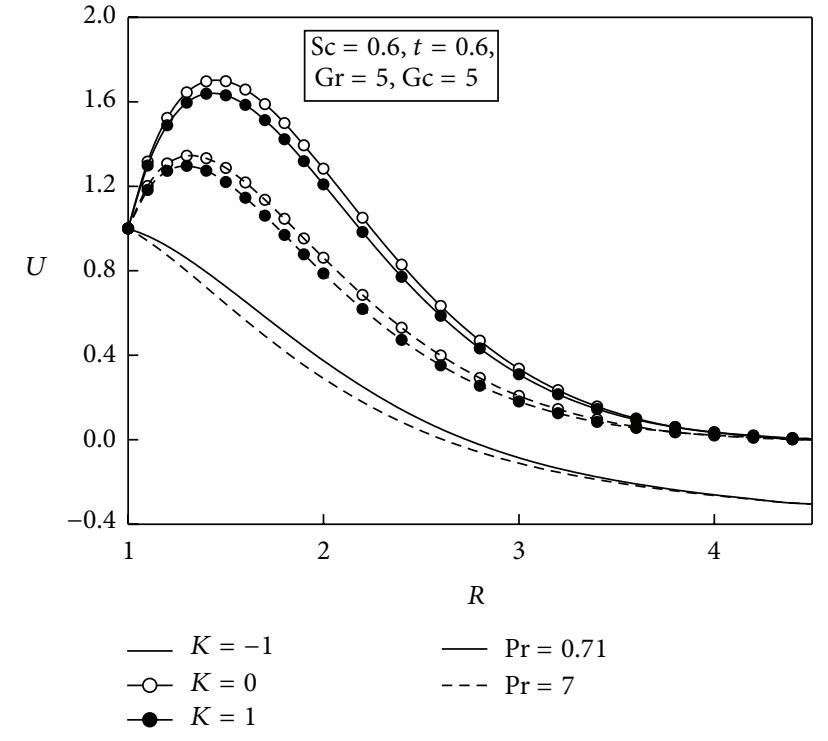

Figure 2: Velocity profiles for different $K$ and $\mathrm{Pr}$ at $\mathrm{Gr}=5, \mathrm{Gc}=5$, $t=0.6$, and $\mathrm{Sc}=0.6$.

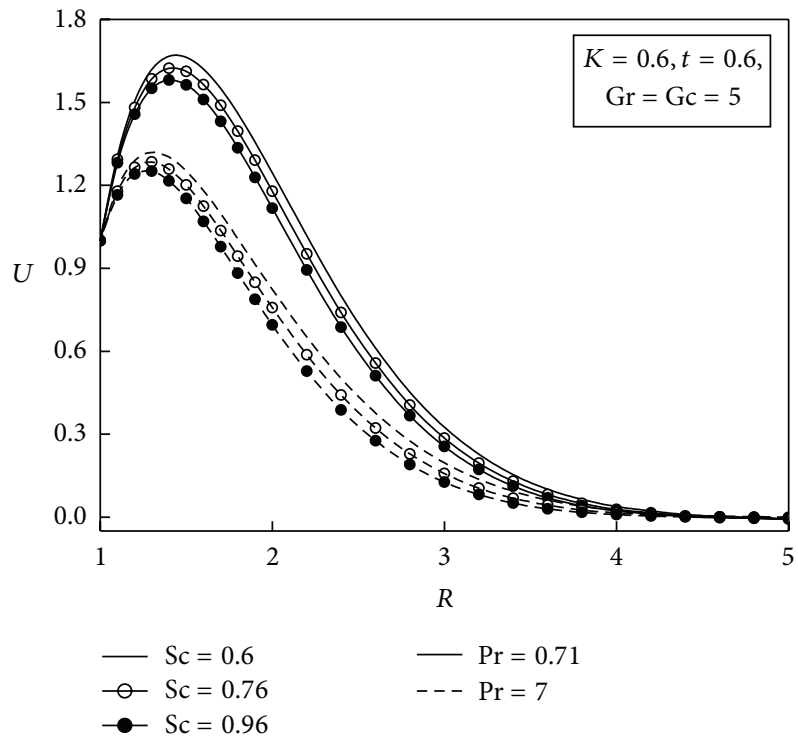

FIGURE 3: Effect of Sc on velocity profiles for $\operatorname{Pr}=0.71$ and 7 at $K=$ $0.6, \mathrm{Gr}=5, \mathrm{Gc}=5$, and $t=0.6$.

\section{Results and Discussions}

In order to understand the physical insight of the problem, numerical computations for the velocity, temperature, concentration, skin friction, Nusselt number, and Sherwood number are made for various physical parameters and presented in Figures 2-11. Since water and air are the most commonly occurring fluids in nature, we basically restricted our observations to $\operatorname{Pr}=0.71$ (air) and $\operatorname{Pr}=7$ (water) only.

Figures 2 and 3 depict the effects of the chemical reaction and Schmidt number on velocity profiles for $\operatorname{Pr}=0.71$ (air) and $\mathrm{Pr}=7$ (water) at $\mathrm{Gr}=5, \mathrm{Gc}=5$, and $t=0.6$. For the case of $K>0$, that is, for destructive reaction, increasing

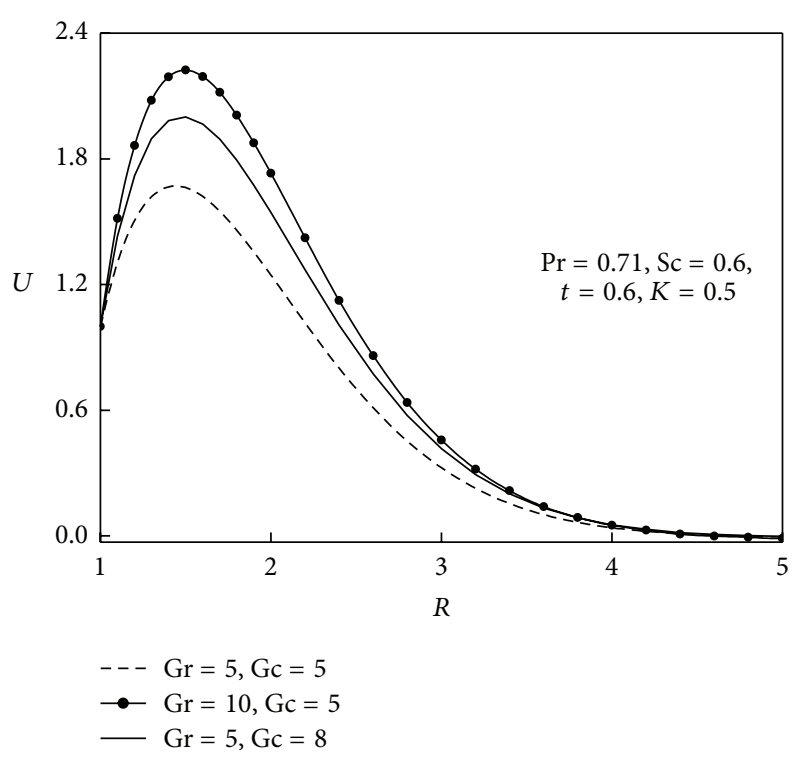

FIGURE 4: Effects of Gr and Gc on velocity profiles at $K=0.5$, Sc $=$ $0.6, t=0.6$, and $\operatorname{Pr}=0.71$.

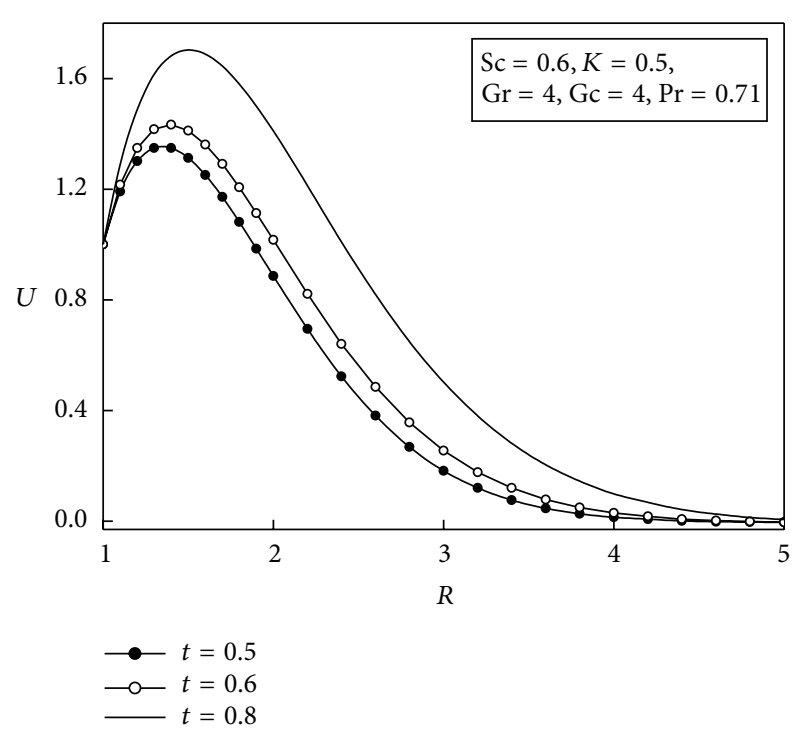

FIGURE 5: Effect of time on velocity profiles at $K=0.5, \mathrm{Gr}=4, \mathrm{Gc}=$ $4, \mathrm{Sc}=0.6$, and $\operatorname{Pr}=0.71$.

values of $K$ lead to a fall in velocity profiles. For generative reaction, $K<0$, a fall in velocity is observed for increasing $K$. This is due to the fact that as $K<0$, the last term in the momentum equation becomes positive and plays a crucial role. Also, velocity profile decreases very fast with generative chemical reaction in comparison with destructive chemical reaction for both air and water. The Schmidt number Sc embodies the ratio of the momentum diffusivity to the species diffusivity. It physically relates the relative thickness of the hydrodynamic boundary layer and concentration boundary layer. It is observed that as the Schmidt number increases, the transient velocity decreases. Effects of the thermal Grashof number (Gr) and mass Grashof number $(\mathrm{Gc})$ at $K=0.5$, 


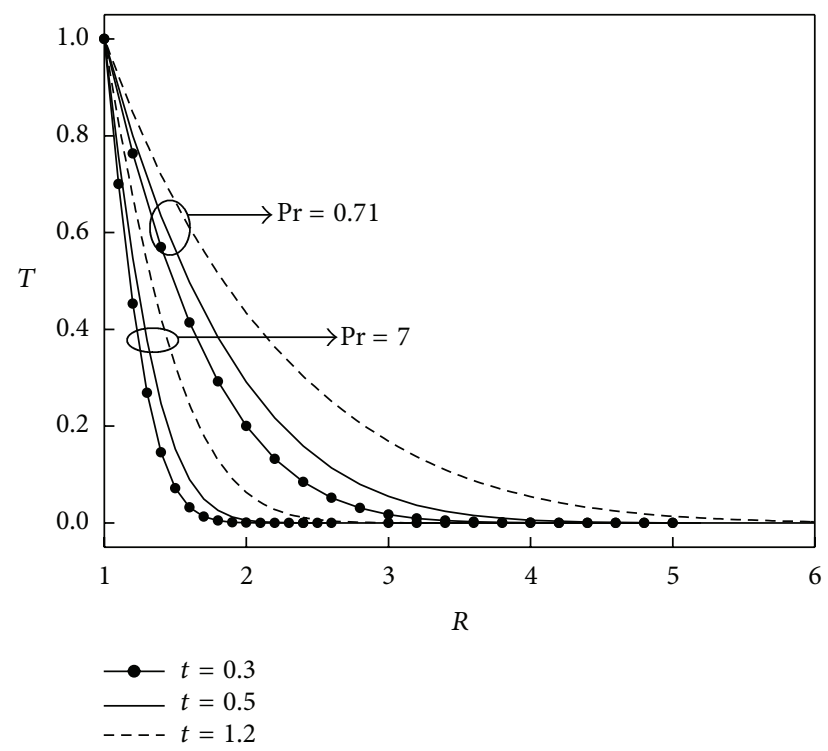

Figure 6: Temperature profiles for different values of the Prandtl number and time.

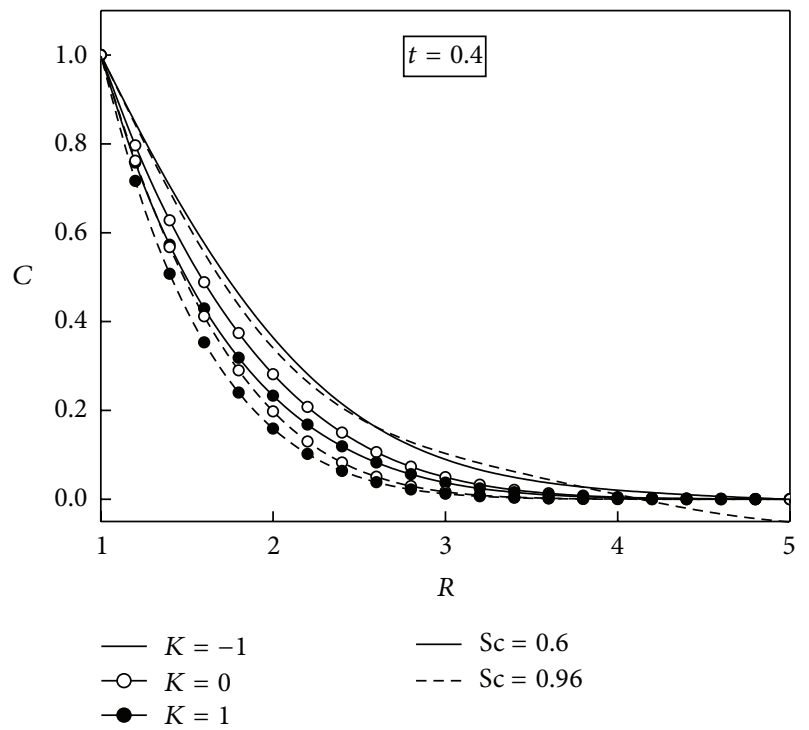

FIGURE 7: Effects of $K$ and Sc on concentration profiles at $t=0.4$.

Sc $=0.6, t=0.6$, and $\operatorname{Pr}=0.71$ are presented in Figure 4 . It is observed that an increase in the thermal Grashof number or mass Grashof number leads to a rapid increase in the fluid velocity. The thermal Grashof number signifies the relative effect of the buoyancy force to the hydrodynamic viscous force. The positive values of $\mathrm{Gr}$ correspond to cooling of the cylinder by natural convection. Heat is, therefore, conducted away from the vertical cylinder into the fluid which increases temperature and thereby enhances the buoyancy force. It is observed that the transient velocity accelerates due to enhancement in the thermal buoyancy force. The solutal Grashof number Gc defines the ratio of the species buoyancy force to the viscous hydrodynamic force. It is noticed that the transient velocity increases considerably with a rise in the

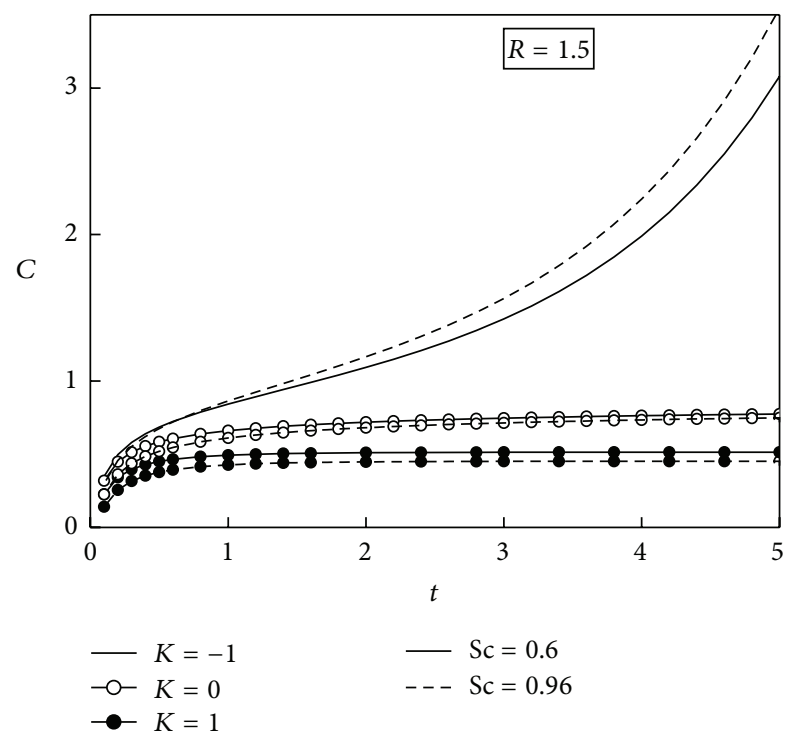

FIGURE 8: Concentration profiles with respect to time for different values of $K$ and Sc at $R=1.5$.

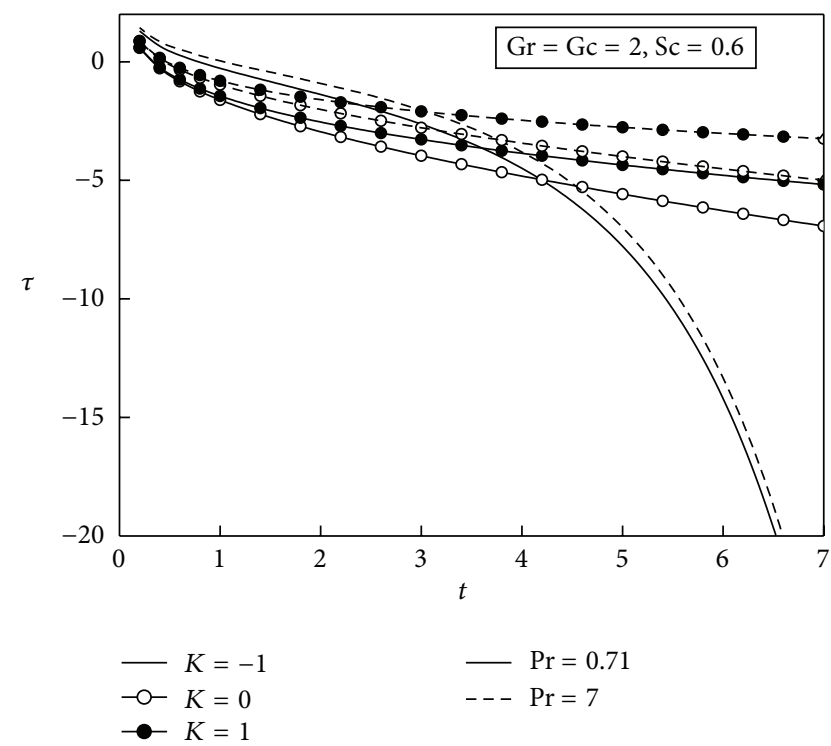

Figure 9: Effects of $K$ and Pr on skin friction at $\mathrm{Gr}=2, \mathrm{Gc}=2$, and $\mathrm{Sc}=0.6$.

species buoyancy force. In both cases, it is interesting to note that as Gr or Gc increases, there is rapid rise in the velocity near the surface of vertical cylinder, and then it descends smoothly to the free stream velocity. Velocity profiles for different values of time at $\mathrm{Gr}=4, \mathrm{Gc}=4, K=0.5$, $\mathrm{Sc}=0.6$, and $\operatorname{Pr}=0.71$ are plotted in Figure 5. It is noticed that fluid velocity increases with increasing time.

The transient temperature profiles for different $\operatorname{Pr}$ and $t$ are plotted in Figure 6. It is noticed that the temperature decreases with an increase in $\operatorname{Pr}$ and increases with an increase in time $t$. The transient concentration profiles for different Schmidt number and chemical reaction parameter are plotted in Figure 7. It is observed from the figure that 


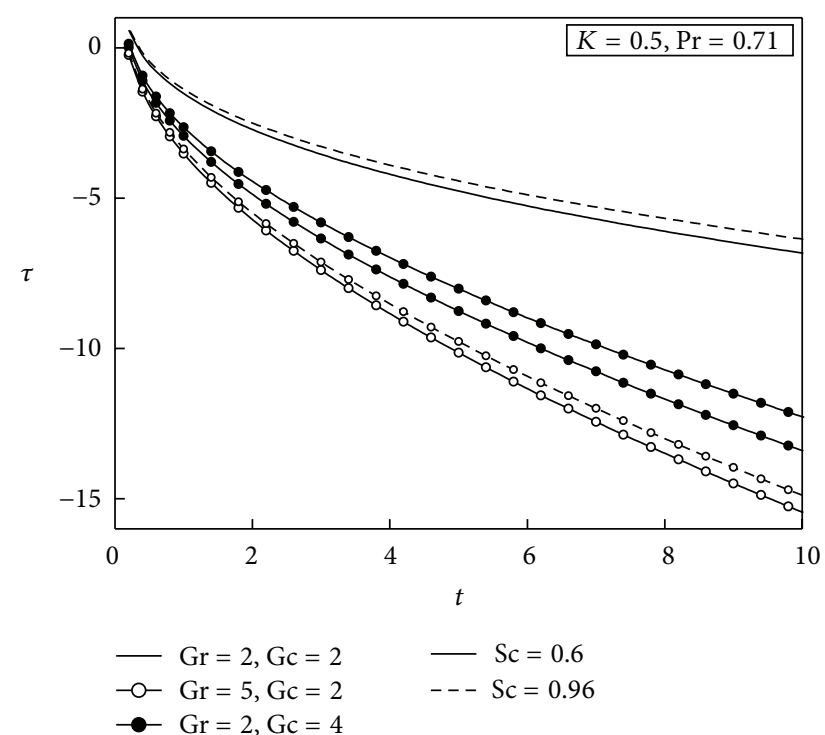

FIGURE 10: Effects of Gr and Gc on skin friction at $K=0.5, \mathrm{Pr}=0.71$, and $\mathrm{Sc}=0.6$ and 0.96 .

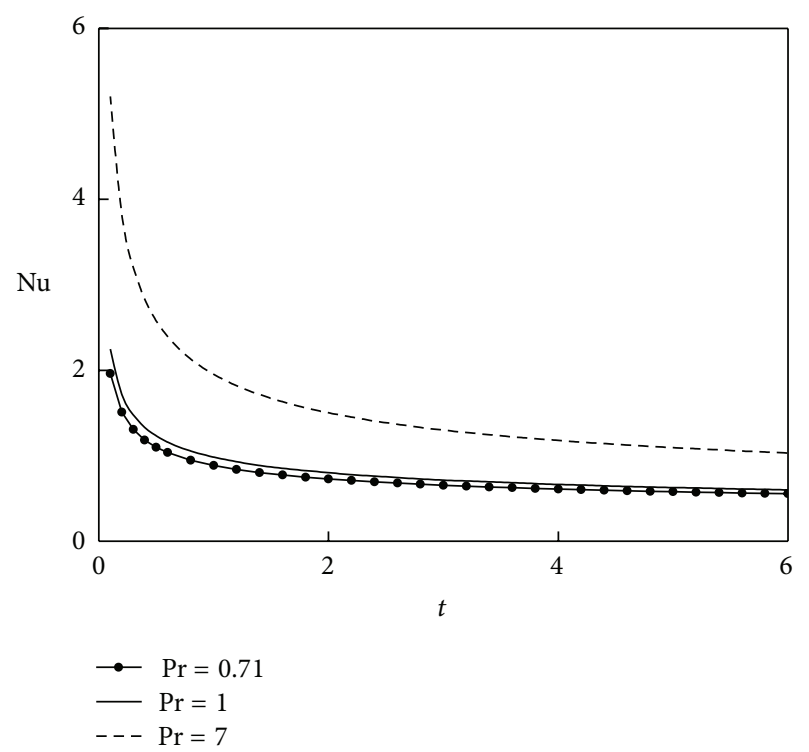

Figure 11: Effects of Pr on the Nusselt number with respect to time.

the concentration decreases with the Schmidt number. For destructive reaction $(K>0)$, as the reaction parameter increases, the concentration profiles decrease. This is due to the fact that as $K>0$, the last term in the mass diffusion equation becomes positive for generative reaction $(K<0)$; the opposite effect is observed.

Figure 8 depicts the concentration profiles for various values of the chemical reaction parameter and Schmidt number with respect to time. It is clear from the figure that, initially, concentration profiles increase with time, but, for larger time, they become steady for destructive chemical reaction or even without chemical reaction. It is interesting to see that for

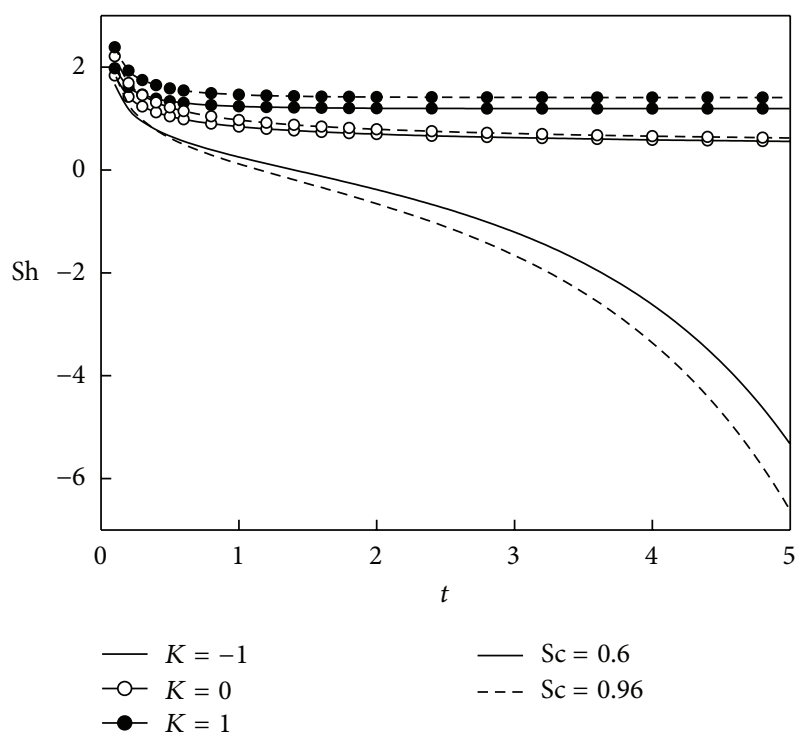

FIGURE 12: Effects of $K$ and Sc on the Sherwood number with respect to time.

generative chemical reaction $(K<0)$, concentration profile increases unboundedly for all values of the Schmidt number.

Figure 9 demonstrates the skin friction against time for various values of the chemical reaction parameter $K$ and Prandtl number $\mathrm{Pr}$ at $\mathrm{Gr}=2, \mathrm{Gc}=2$, and $\mathrm{Sc}=0.6$, which reflects that skin friction increases with the destructive chemical reaction $(K>0)$ or Prandtl number. It is noticeable that for generative chemical reaction $(K<0)$, the skin friction shows an opposite character and decreases as time progresses. Effects of the thermal Grashof number, mass Grashof number, and Schmidt number are plotted in Figure 10. It is observed that an increase in the value of the Schmidt number increases skin friction, whereas it decreases with the increase in the value of the thermal Grashof number or mass Grashof number.

Figure 11 depicts the effects of the Prandtl number on rate of heat transfer with respect to time, which shows that, initially, the Nusselt number decreases sharply with time and tends to steady at larger time. Also, the Nusselt number increases with increase in the Prandtl number.

Effects of the chemical reaction parameter and Schmidt number on the Sherwood number are depicted in Figure 12. It can be observed from the figure that, initially, the Sherwood number falls sharply with time but tends to steady state for destructive chemical reaction or no chemical reaction, whereas, for generative chemical reaction $(K<0)$, the Sherwood number decreases unboundedly. Also, the Sherwood number increases with increased values of the Schmidt number.

\section{Conclusions}

In this paper, the authors have analyzed the effect of first order chemical reaction on unsteady free convection flow past an infinite vertical cylinder with combined heat and mass 
transfer. The closed-form solutions of the governing partial differential equations are obtained by the Laplace transform technique. Effects of various physical parameters involved in the flow problem are also analyzed and discussed with graphical results. Based on their observations and discussions above, they summarize their conclusions as follows.

(i) Transient velocity decreases with chemical reaction. Velocity profile increases with increase in buoyancy force parameters but decreases with increase in the Prandtl number or Schmidt number.

(ii) Transient temperature profiles increase with the Prandtl number.

(iii) Transient concentration profile decreases with the Schmidt number and destructive chemical reaction but increases with generative chemical reaction.

(iv) Skin friction increases with the destructive chemical reaction or Schmidt number but decreases with the thermal and mass Grashof number.

(v) The Nusselt number increases with the Prandtl number.

(vi) The Sherwood number increases with increase in destructive chemical reaction and tends to steady state at larger time, while, for generative chemical reaction, there is a decrease in the Sherwood number.

\section{Nomenclature}

$C^{\prime}$ : Concentration

C: Dimensionless concentration

$D$ : Mass diffusion coefficient

Gr: The thermal Grashof number

Gc: The mass Grashof number

$g$ : Acceleration due to gravity

$J_{0}$ : Bessel's function of the first kind and order zero

$J_{1}$ : Bessel's function of the first kind and order one

$k_{1}$ : Chemical reaction parameter

$K$ : Dimensionless chemical reaction parameter

$K_{0}$ : Modified Bessel's function of the second kind and order zero

$K_{1}$ : Modified Bessel's function of the second kind and order one

$\mathrm{Nu}$ : The Nusselt number

Pr: The Prandtl number

$r$ : Radial coordinate measured from the axis of the cylinder

$R$ : Dimensionless radial coordinate

Sc: The Schmidt number

Sh: The Sherwood number

$t^{\prime}$ : Time

$t$ : Dimensionless time

$T^{\prime}$ : Temperature of fluid

$T$ : Dimensionless fluid temperature

$u$ : Vertical velocity
$U$ : Dimensionless vertical velocity

$Y_{0}$ : Bessel's function of the second kind and order zero

$Y_{1}$ : Bessel's function of the second kind and order one.

\section{Greek Symbols}

$\alpha$ : Thermal diffusivity of fluid

$\nu$ : Kinematic viscosity

$\beta$ : Volumetric coefficient of thermal expansion

$\beta^{*}$ : Volumetric coefficient of expansion with concentration.

\section{References}

[1] E. M. Sparrow and J. L. Gregg, "Laminar free convection heat transfer from the outer surface of a vertical circular cylinder," Transactions of ASME, vol. 78, pp. 1823-1829, 1956.

[2] R. J. Goldstein and D. G. Briggs, "Transient free convection about vertical plates and circular cylinders," Journal of Heat Transfer, vol. 86, pp. 490-500, 1964.

[3] A. Ishak, "Mixed convection boundary layer flow over a vertical cylinder with prescribed surface heat flux," Journal of Physics A, vol. 42, no. 19, Article ID 195501, 2009.

[4] F. S. Lien, C. K. Chen, and Y. M. Chang, "Effects of free convection and mass transfer on the flow past an impulsively moving infinite vertical circular cylinder," International Communications in Heat and Mass Transfer, vol. 12, no. 2, pp. 127-137, 1985.

[5] P. L. Chambré and J. D. Young, "On the diffusion of a chemically reactive species in a laminar boundary layer flow," Physics of Fluids, vol. 1, no. 1, pp. 48-54, 1958.

[6] U. N. Das, R. Deka, and V. M. Soundalgekar, "Effects of mass transfer on flow past an impulsively started infinite vertical plate with constant heat flux and chemical reaction," Engineering Research, vol. 60, no. 10, pp. 284-287, 1994.

[7] R. Muthucumaraswamy and P. Ganesan, "First-order chemical reaction on flow past an impulsively started vertical plate with uniform heat and mass flux," Acta Mechanica, vol. 147, no. 1-4, pp. 45-57, 2001.

[8] K. Gangadhar, N. B. Reddy, and P. K. Kameswaran, "Similarity solution of hydro magnetic heat and mass transfer over a vertical plate with convective surface boundary condition and chemical reaction," International Journal of Nonlinear Science, vol. 3, no. 3, pp. 298-307, 2012.

[9] P. Ganesan and H. P. Rani, "On diffusion of chemically reactive species in convective flow along a vertical cylinder," Chemical Engineering and Processing, vol. 39, no. 2, pp. 93-105, 2000.

[10] P. Ganesan and P. Loganathan, "Heat and mass flux effects on a moving vertical cylinder with chemically reactive species diffusion," Journal of Engineering Physics and Thermophysics, vol. 75, no. 4, pp. 899-909, 2002.

[11] H. P. Rani, G. J. Reddy, and C. N. Kim, "Transient analysis of diffusive chemical reactive species for couple stress fluid flow over vertical cylinder," Applied Mathematics and Mechanics, vol. 34, no. 8, pp. 985-1000, 2013. 
[12] G. R. Machireddy, "Chemically reactive species and radiation effects on MHD convective flow past a moving vertical cylinder," Ain Shams Engineering Journal, 2013.

[13] H. S. Carslaw and J. C. Jaeger, Operational Methods in Applied Mathematics, Oxford Press, Oxford, UK, 2nd edition, 1948. 

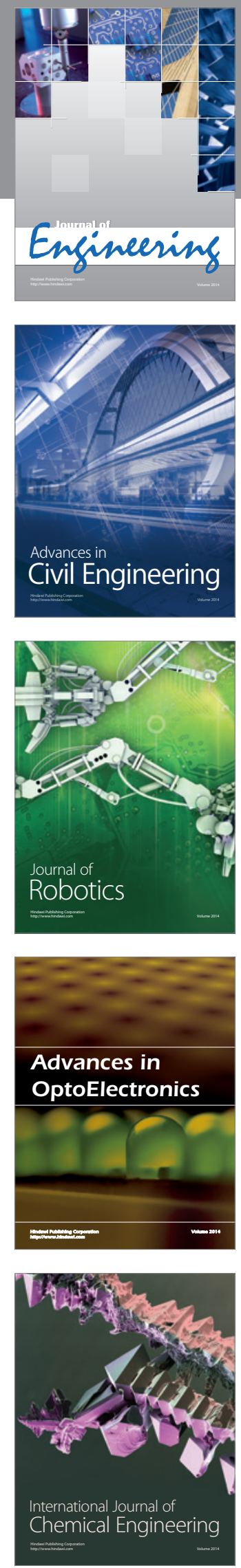

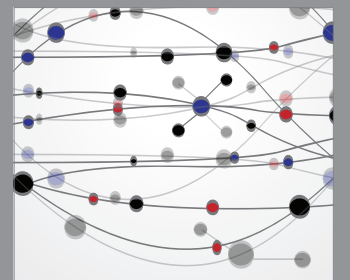

The Scientific World Journal
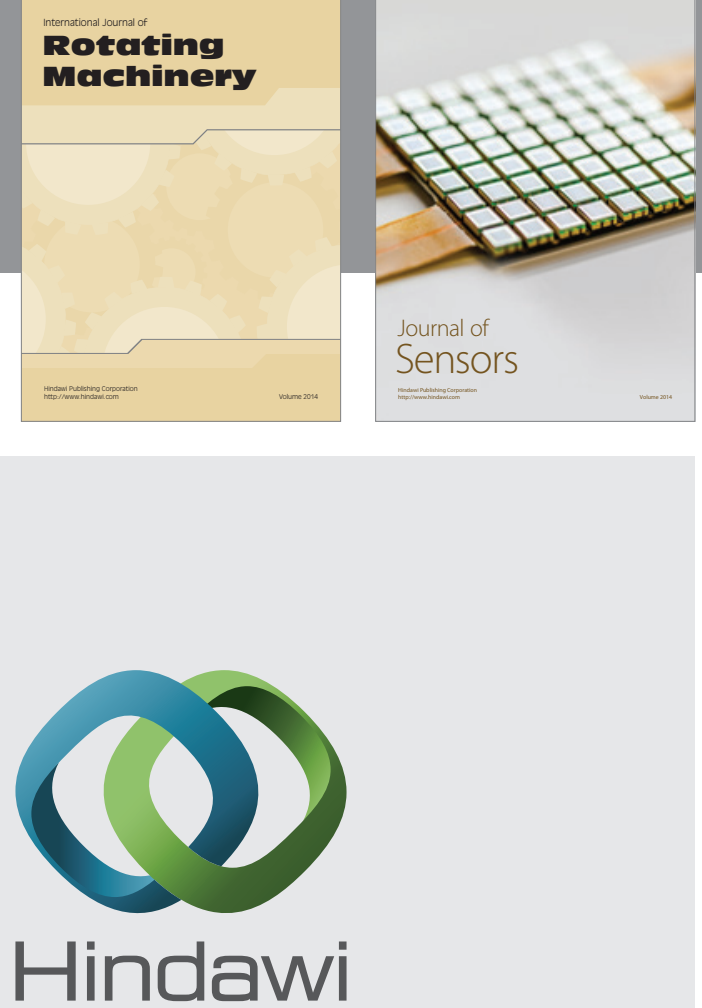

Submit your manuscripts at http://www.hindawi.com
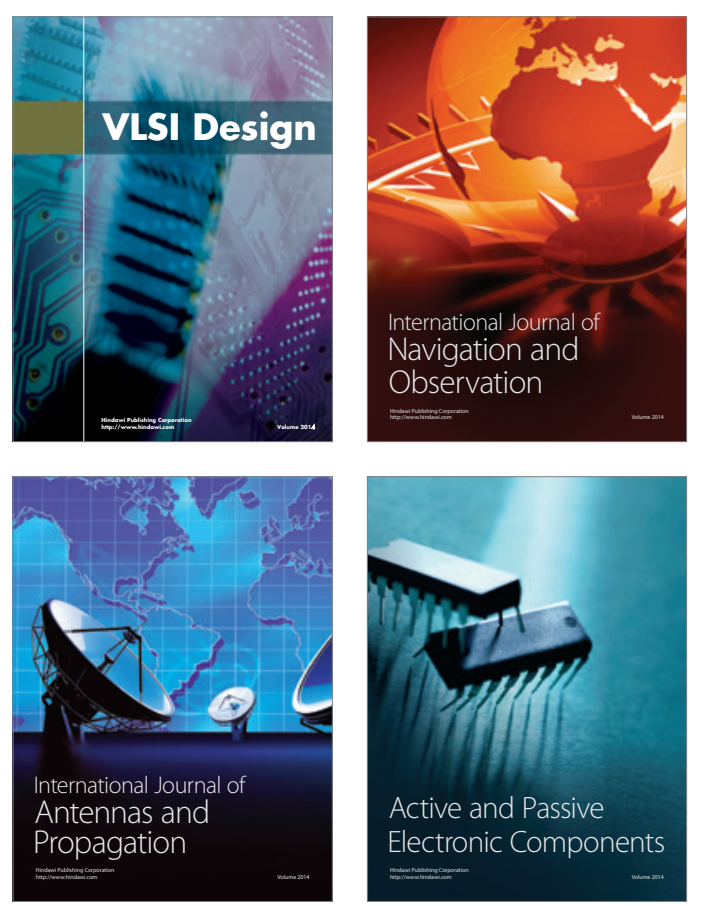
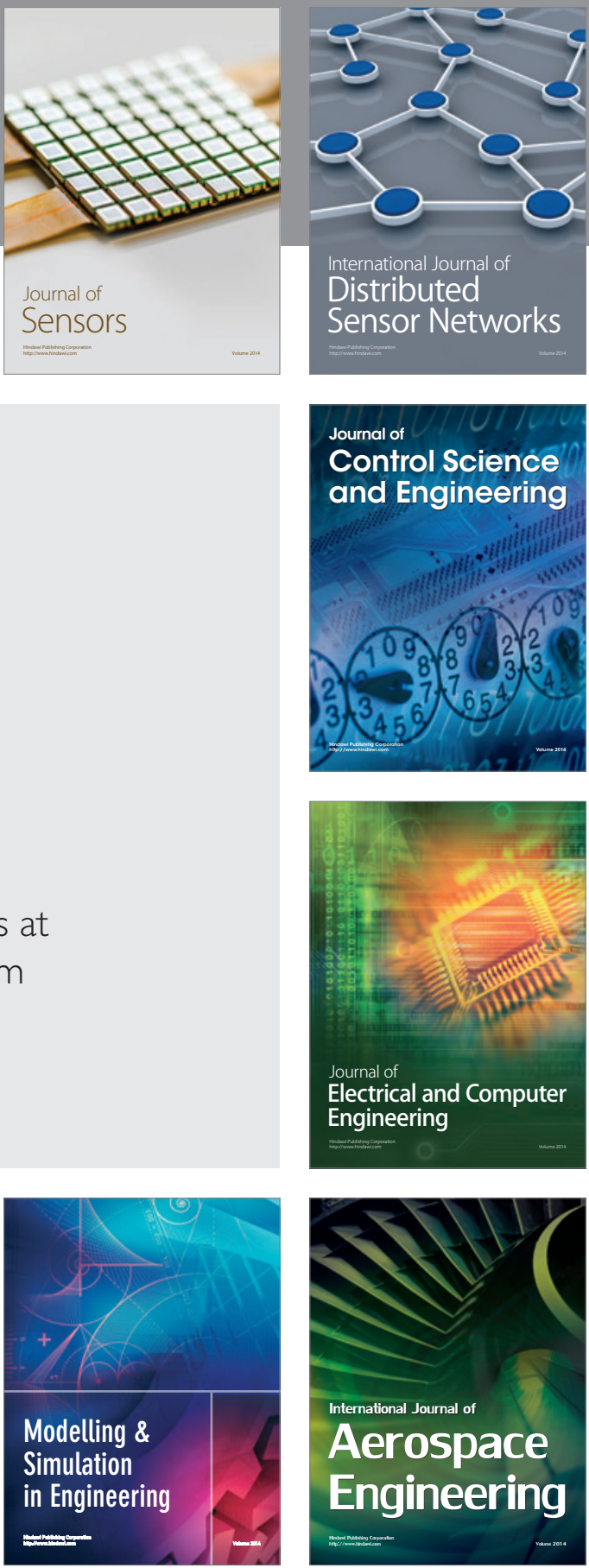

Journal of

Control Science

and Engineering
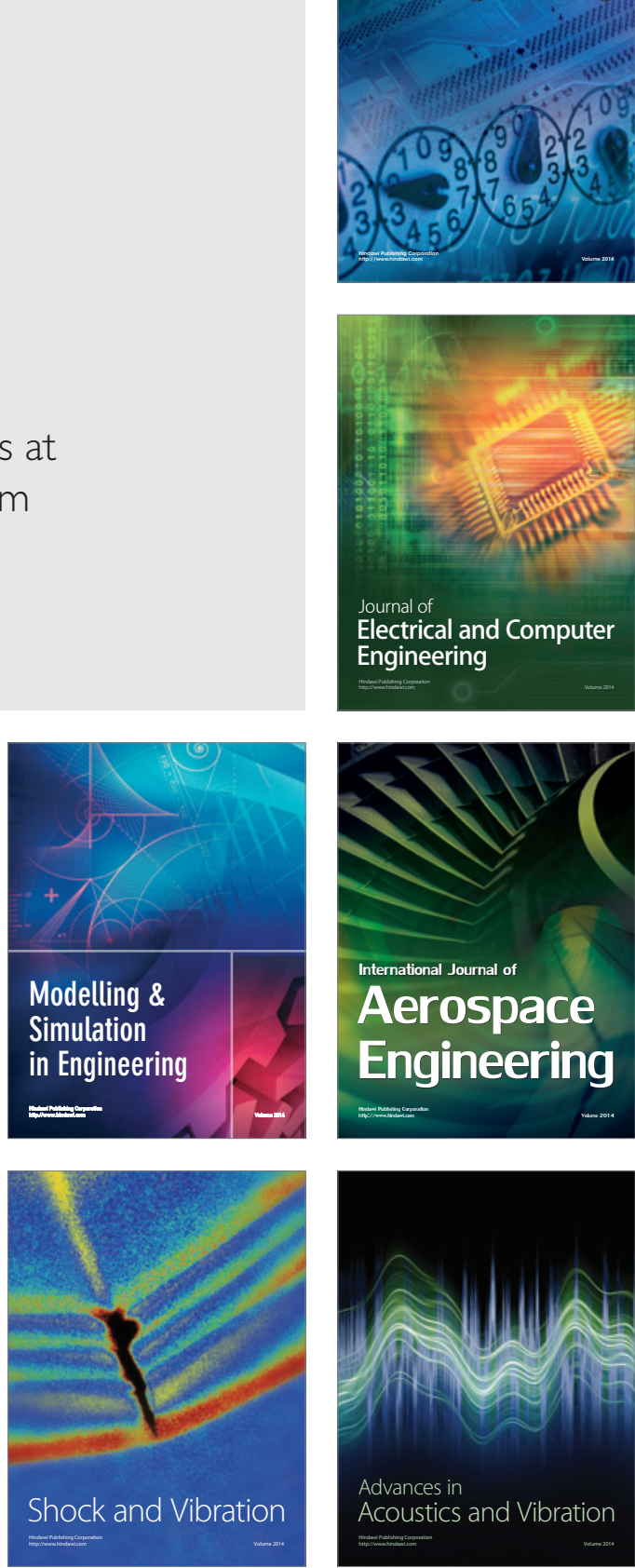